\title{
The early but not the late neural correlate of auditory awareness reflects lateralized experiences
}

\author{
Rasmus Eklund ${ }^{a, *}$, Billy Gerdfeldter ${ }^{a}$ and Stefan Wiens ${ }^{a}$ \\ ${ }^{a}$ Gösta Ekmans Laboratorium, Stockholm University
}

\section{ARTICLE INFO}

\section{Keywords:}

auditory

consciousness

auditory awareness negativity

late positivity

lateralization

\begin{abstract}
A B S T R ACT
Theories disagree as to whether it is the early or the late neural correlate of awareness that plays a critical role in phenomenal awareness. According to recurrent processing theory, early activity in primary sensory areas corresponds closely to phenomenal awareness. In support, research with electroencephalography found that in the visual and somatosensory modality, an early neural correlate of awareness is contralateral to the perceived side of stimulation. Thus, early activity is sensitive to the perceived side of visual and somatosensory stimulation. Critically, it is unresolved whether this is true also for hearing. In the present study ( $N=26$ students), Bayesian analyses showed that the early neural correlate of awareness (auditory awareness negativity, AAN) was stronger for contralateral than ipsilateral electrodes and moderate evidence that the late correlate of auditory awareness (late positivity, LP) was not lateralized. These findings demonstrate that the early but not the late neural correlate of auditory awareness reflects lateralized experiences. Thus, the findings support that early and late neural correlates capture separate aspects of consciousness.
\end{abstract}

\section{Introduction}

Theories disagree on how neural activity generates awareness. The recurrent processing theory (Lamme, 2006) suggests that: (1) early feedforward activity from sensory stimulation is an unconscious process, (2) early local feedback loops between the primary sensory cortices and hierarchically higher areas generate the subjective sensation of what an experience is like (phenomenal awareness), and (3) later, global feedback loops enable introspection and reporting of the experience (access consciousness). In contrast, the global neuronal workspace theory (Dehaene et al., 2006) suggests that early recurrent processing is preconscious and that only late, global activity mediates consciousness.

Neural activity that is associated with awareness is commonly referred to as the neural correlate of consciousness (NCC; Crick and Koch, 1990). One common method of identifying the NCC is through a contrastive analysis: The same stimulus is presented over multiple trials, and the NCC is the difference in neural activity between trials rated as aware and trials rated as unaware (Aru et al., 2012).

Research on NCC has focused mainly on vision (Koivisto and Revonsuo, 2010), whereas research on hearing and touch has been sparser (Dykstra et al., 2017; Meyer, 2011; Snyder et al., 2015). The NCC has been extensively studied with electroencephalography (EEG) and magnetoencephalography (MEG) because of their excellent temporal resolution.

Two common NCC have been identified. In EEG, these appear as an early (at about $200 \mathrm{~ms}$ ) negativity and a late (after $300 \mathrm{~ms}$ ) centroparietal positivity (late positivity, LP). The early NCC is referred to as visual awareness negativity (VAN) in vision (Koivisto and Revonsuo, 2010) and as auditory awareness negativity (AAN) in hearing (Eklund and

\footnotetext{
*Corresponding author

@ rasmus.eklundepsychology.su.se (R. Eklund)

ORCID(s): 0000-0001-9924-5486 (R. Eklund); 0000-0002-3222-8056 (B. Gerdfeldter); 0000-0003-4531-4313 (S. Wiens)
}

\section{Wiens, 2019).}

Because the early NCC is localized to sensory areas (Andersen et al., 2016; Eklund et al., 2020; Eklund and Wiens, 2019; Forschack et al., 2020; Koivisto and Revonsuo, 2010) and involves recurrent processing (Auksztulewicz and Blankenburg, 2013; Boehler et al., 2008), these findings support recurrent processing theory. Furthermore, whereas global neuronal workspace theory emphasizes the role of late activity (LP) in awareness, recurrent processing theory postulates that LP reflects only post-perceptual processing. In support, LP is strongly affected by response manipulations (e.g., Förster et al., 2020; Pitts et al., 2018; Tsuchiya et al., 2015; Dembski et al., 2021).

In further support of the idea that the early NCC matches phenomenal experience, the location of early processing in sensory areas corresponds to the perceived side of stimulation. Specifically, the visual awareness negativity is contralateral to visual stimulation (Boehler et al., 2008; Eklund and Wiens, 2018; Koivisto and Grassini, 2016), and the early NCC of touch is contralateral to tactile stimulation (Auksztulewicz and Blankenburg, 2013; Forschack et al., 2020). Notably, this research suggests that the late NCC in vision and touch are not lateralized (as suggested by their scalp topographies).

In hearing, previous research does not clarify whether early and late NCC are lateralized. Whereas the visual and somatosensory pathways are clearly lateralized contralaterally, the auditory pathway afferently bifurcates and decussates at several junctions (Pickles, 2015). As a result, the NCC in hearing may not be as clearly lateralized as the NCC in vision and touch.

Although many studies have investigated neural responses to lateralized auditory stimulation in various contexts, this research did not explicitly examine awareness (e.g., Butcher et al., 2011; Gutschalk and Steinmann, 2015; Virtanen et al., 1998; Woldorff et al., 1999). Furthermore, research that has 
investigated activity more closely related to awareness does not lend itself to resolving this question, for one or more of the following reasons (Alain et al., 2001; Hautus and Johnson, 2005; Königs and Gutschalk, 2012; Sanders et al., 2008): First, because a contrastive analysis was not used, the NCC could not be isolated. Second, it is unclear whether lateralized perception was captured. Third, because trials rated as aware and unaware differed physically, the NCC may have been confounded by physical stimulus differences. Fourth, because stimuli were presented only on one side, a contralateral response that was stronger than the simultaneous ipsilateral activity could simply reflect that one hemisphere responds more strongly.

Here, we examined whether the early NCC (AAN) and the late NCC (LP) in hearing are lateralized by playing soft clicks heard to the left or right side. The main hypotheses of the study were that AAN is lateralized and that LP is not. Specifically, AAN should be stronger for electrodes that are contralateral to auditory stimulation than for electrodes that are ipsilateral to auditory stimulation.

The experiment was designed to test these hypotheses while avoiding the aforementioned issues. First, we used a contrastive analysis. Second, auditory stimuli were lateralized binaural clicks that subjects perceived as a single, lateralized click. Third, AAN and LP were computed to physically identical stimuli. Fourth, AAN and LP were measured to both left and right clicks. Furthermore, the hypotheses, method, and analyses were preregistered to minimize potential biases from any flexibility in data processing (Luck and Gaspelin, 2017). Bayesian hypothesis testing (Dienes and McLatchie, 2018) was used to assess the strength of evidence that AAN is lateralized and that LP is not. Bayesian hypothesis testing is preferable to null hypothesis significance testing because it quantifies the strength of evidence for (or against) a predicted effect (Wagenmakers et al., 2016).

\section{Method}

We preregistered the primary hypotheses, methods, and analyses (https://doi.org/10.17605/OSF. IO/PSRJF) to avoid bias (Luck and Gaspelin, 2017; Nosek et al., 2018). Supplementary material is available at a university repository (Wiens et al., 2021).

\subsection{Participants}

The rationale for sample size and recruitment was similar as in our previous study (Eklund et al., 2020). Because departmental regulations restricted subject recruitment because of COVID-19, data collection was extended until October 2020. The sample consisted of 28 student volunteers (12 male; 25 right-handed; age: $M=27.5, S D=3.1$ ). Ethical review and approval were not required for the study on human participants in accordance with local law and institutional requirements. The experiment adhered to the declaration of Helsinki. Participants provided their written informed consent to participate in this study and that their raw data will be shared anonymized.
We deviated from the preregistration by excluding two subjects who lacked trials (less than 5) in one of the critical conditions. Without these trials, it was not possible to conduct the preregistered analyses. The final sample for the preregistered analyses consisted of 26 subjects (12 male; 23 right-handed; age: $M=27.9, S D=3.0$ ).

\subsection{Apparatus and stimuli}

Stimuli were auditory clicks $(4 \mathrm{~ms})$ lateralized to the left or right ear (stimulus EE1 from Thaler et al., 2017). Lateralization was manipulated with an interaural time difference $( \pm 0.25 \mathrm{~ms})$ and an interaural level difference $( \pm 8 \mathrm{~dB})$. Clicks were presented through in-ear tubephones (ER2; Etymotic Research Inc., IL; www. etymotic. com). Instructions were displayed on a BenQ XL2430T, 24-inch gaming monitor (at $144 \mathrm{~Hz}, 1920 \times 1080$ resolution). PsychoPy v 3.0 (Peirce, 2007) was used to run the experiment and to collect behavioral data.

Awareness was measured with the perceptual awareness scale (Sandberg et al., 2010). The almost clear rating was excluded, as in our previous research (Eklund and Wiens, 2019; Eklund et al., 2020; Eklund and Wiens, 2018).

\subsection{Procedure}

The procedure resembled that of our previous study (Eklund et al., 2020). Subjects performed a detection and discrimination task that comprised 800 trials ( 720 critical and 80 catch). Critical trials contained a click that was lateralized to either left or right and was presented at the individual auditory awareness threshold (see below). Catch trials did not contain a click. The trials were divided into eight blocks. Each block comprised 100 trials (90 critical and 10 catch). Within each set of 20 trials ( 9 left, 9 right, and 2 catch trials), trial order was randomized. There was a short break between blocks.

Figure 1 shows the time course of a trial. On each trial, a fixation cross was shown for $1000 \mathrm{~ms}$. On critical trials, a left or right click was played $500 \mathrm{~ms}$ after trial onset. After each trial, subjects first indicated whether they heard a left or right click by using one of two buttons ("left arrow"

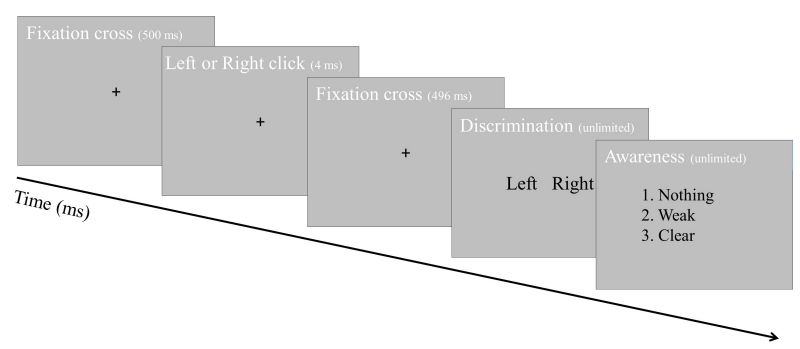

Figure 1: The time course of a trial. On each trial, a black fixation cross $\left(0.5^{\circ}\right)$ was displayed on a black background for $1000 \mathrm{~ms}$. On each critical trial, a click (left or right lateralized) was played binaurally $500 \mathrm{~ms}$ after trial onset. On catch trials, no click was played. Afterward, subjects discriminated click side (left or right) and rated their subjective awareness of the click. 
and "right arrow," corresponding to left click and right click, respectively). Afterward, they rated their subjective awareness of the click by using one of three buttons ("1", " 2 ", and "3," corresponding to "I did not hear a click," "I heard the click weakly," and "I heard the click clearly," respectively). Subjects were instructed to evenly distribute their responses (left and right) when they did not hear the click to discourage using a strategy in the discrimination task (e.g., always guessing left click when not hearing the click). Subjects were also instructed to focus on rating their awareness accurately rather than responding quickly.

Before the experiment, subjects familiarized themselves with the experimental procedure through a short practice session; this session was identical to the experiment but with clearly audible clicks. Then, psychophysical staircases were used to calibrate the left and right click separately to an intensity level at which an individual subject reported hearing the click (i.e, rated it as weakly or clearly audible) on approximately $50 \%$ of the trials; this level was designated the individual auditory awareness threshold for each click side. The staircase procedure and the calibration procedure was similar to that in our previous study (Eklund et al., 2020) except that each procedure contained 45 critical trials per click side.

\subsection{EEG recording}

The EEG recording was identical to that of our previous study (Eklund et al., 2020). EEG data were recorded from 64 electrodes at standard 10-20 position with an Active Two BioSemi system (BioSemi, Amsterdam, Netherlands). An EEG cap (Electro-Cap International, Eaton, OH) was used to position these electrodes together with system-specific electrodes (https://www. biosemi.com/faq/cms\&drl.htm). Two additional positions (tip of the nose and right cheek) were recorded Data were sampled at $1024 \mathrm{~Hz}$ and filtered with a hardware low-pass filter at $208 \mathrm{~Hz}$.

\subsection{EEG preprocessing}

As in our previous study (Eklund et al., 2020), the data from the 64 standard electrode positions, together with the tip of the nose and the right cheek, were processed and analyzed offline with MNE-Python (Gramfort et al., 2013, 2014; Appelhoff et al., 2019; Pernet et al., 2019). The behavioral analyses included all trials, whereas in the EEG data analyses, some trials were excluded (see below). The continuous EEG data were processed offline. Although it was not mentioned in the preregistration, we downsampled the data to $256 \mathrm{~Hz}$ to speed up preprocessing. For each subject, a 1-Hz high-pass filter was applied to remove slow drift, and the electrodes were visually inspected to detect noisy electrodes. Only a few electrodes had to be interpolated (spherical spline interpolation) from neighboring electrodes $(M=$ $0.38, S D=0.74)$. For the independent component analysis, noisy channels were interpolated and eye blinks were automatically detected from the eye blink channel (i.e., Fpz vs. cheek). Independent component analysis (fastica) was conducted, and eye blink components were removed $(M=1.50$,
$S D=0.64$ ) on the basis of their topography and their correlation with the eye blink channel. Afterward, the data were re-referenced to the tip of the nose.

Epochs were extracted from $100 \mathrm{~ms}$ before tone onset to $600 \mathrm{~ms}$ after tone onset. Each epoch was baseline corrected to the mean of the 100-ms interval before tone onset $(-100$ to $0 \mathrm{~ms}$ ). For each subject, maximum amplitude ranges were extracted for individual epochs (after a 30-Hz low-pass filter), and the distribution of these amplitude ranges was inspected for outliers. The number of outliers removed per subject was $M=11.54(S D=11.19)$, corresponding to $M=$ $1.44 \%(S D=1.40)$. The exclusion thresholds were set for each individual because subjects showed substantial variability in these amplitude ranges. Critically, inspection of trials was blinded to trial type (critical, catch), click side, identification response, and awareness ratings to avoid bias (Keil et al., 2014).

\subsection{EEG analysis}

As preregistered, aware trials were clicks rated as "I heard the click clearly" or "I heard the click weakly," whereas unaware trials were clicks rated as "I did not hear a click." The AAN-relevant interval was the period between 160 and 260 ms after stimulus onset, and the LP-relevant interval was the period between 350 and $550 \mathrm{~ms}$. The electrodes designated as left were F7, F5, F3, F1, FT7, FC5, FC3, FC1, T7, C5, C3, C1, TP7, CP5, CP3, and CP1. The electrodes designated as right were F8, F6, F4, F2, FT8, FC6, FC4, FC2, T8, C6, $\mathrm{C} 4, \mathrm{C} 2$, TP8, CP6, CP4, and CP2. These intervals and this set of electrodes were most sensitive to AAN and LP in our previous studies (Eklund et al., 2020, 2019).

Difference waves were calculated from correctly lateralized trials rated as aware (aware correct) minus correctly and . incorrectly lateralized trials rated as unaware. Thus, these difference waves represented the NCC. On some trials, subjects reported awareness of the click on the wrong side. On these aware incorrect trials, subjects may have pressed the wrong button (lapse trial), or the response may have actually reflected their experience (Eklund et al., 2020). Although aware trials could be grouped according to the experienced laterality, this would introduce a physical confound in the contrastive analysis for each click side. Specifically, trials rated as aware would include clicks from both sides, whereas trials rated as unaware would include clicks from only one side. Therefore, aware incorrect trials were excluded.

Four difference waves were computed as defined by the combination of click side (left or right) and electrode laterality (contralateral or ipsilateral). For example, left contralateral reflected the NCC to a left click across right-sided electrodes, whereas left ipsilateral reflected the NCC to a left click across left-sided electrodes.

As a manipulation check for an overall AAN and overall LP, mean amplitudes were computed across the four difference waves. For AAN (recorded between 160 and 260 ms), we predicted a negative mean amplitude across electrodes. For LP (recorded between 350 and $550 \mathrm{~ms}$ ), we predicted a positive mean amplitude across electrodes. 
Table 1

Descriptive statistics (Mean and SD) of awareness, separately for left and right clicks ( $N$ $=26)$

\begin{tabular}{|c|c|c|c|c|c|c|}
\hline & \multicolumn{2}{|c|}{ Left clicks } & \multicolumn{2}{|c|}{ Right clicks } & \multicolumn{2}{|c|}{ Difference } \\
\hline & Mean & $S D$ & Mean & $S D$ & Mean & $95 \% \mathrm{Cl}$ \\
\hline Aware correct $(\%)$ & 46.0 & 14.3 & 47.1 & 16.1 & -1.1 & {$[-7.3,5.2]$} \\
\hline Aware incorrect (\%) & 6.3 & 6.1 & 4.8 & 6.1 & 1.5 & {$[-0.9,3.8]$} \\
\hline Unaware correct $(\%)$ & 26.1 & 10.3 & 25.8 & 8.0 & 0.2 & {$[-4.3,4.8]$} \\
\hline Unaware incorrect (\%) & 21.6 & 8.6 & 22.3 & 9.4 & -0.7 & {$[-5.5,4.2]$} \\
\hline
\end{tabular}

Note. Percentages refer to the number of trials for each click side (thus, the means for each side sum to 100 ).

To examine whether AAN and LP were lateralized, a new difference wave was calculated from the mean of the two contralateral difference waves (i.e., left contralateral and right contralateral) and the mean of the two ipsilateral difference waves (i.e., left ipsilateral and right ipsilateral). If AAN is lateralized, this difference should be negative between 160 and $260 \mathrm{~ms}$ after stimulus onset. If LP is lateralized, this difference should be positive between 350 and $550 \mathrm{~ms}$.

As preregistered and similar to our previous study, the Bayes Factor $(B F)$ was computed from Bayesian one-sample $t$ tests of difference scores (Dienes and McLatchie, 2018). A $B F_{10}>1$ implies that there is more evidence for the alternative hypothesis than the null hypothesis, whereas $B F_{01}>$ 1 implies that there is more evidence for the null hypothesis than the alternative hypothesis. We used an interpretation scheme of the continuous $B F$ to facilitate verbal communication (Wagenmakers et al., 2018). The alternative hypotheses were modeled as uniform distributions $(-1 \mu \mathrm{V}$ to $+1 \mu \mathrm{V})$, and the likelihood was modeled as a $T$ distribution. We also report the $95 \%$ confidence intervals for mean amplitudes.

\section{Results}

\subsection{Behavior}

Table 1 shows the descriptive statistics for the behavioral data $(N=26)$. Clicks were presented close to the individual awareness threshold (50\%) because close to $50 \%$ of the critical left and right clicks were rated as aware. Of the clicks rated as aware, most were rated as weakly heard $(M=97.8 \%$, $S D=9.3)$. The mean false alarm rate was $12.1 \%(S D=$ 11.5).

\subsection{Electrophysiology}

Figure 2 shows the grand mean waves for individual combinations of click side and electrode laterality for aware trials, unaware trials, and their difference. Figures $3 \mathrm{~A}$ and $\mathrm{B}$ show the grand mean difference waves (aware minus unaware) for left and right clicks for contralateral (solid) and ipsilateral (dotted) electrodes. All difference waves show a negativity peaking at around $200 \mathrm{~ms}$ after click onset (i.e., AAN) and a positivity peaking at around $400 \mathrm{~ms}$ after click onset (i.e., LP). Figure $3 \mathrm{C}$ shows topographies of AAN and LP. Figures $3 \mathrm{~A}$ and $\mathrm{B}$ suggest that that only AAN is stronger for contralateral than ipsilateral electrodes.

In support, Table 2 shows the descriptive and inferential statistics for the preregistered analyses. Figure 4 shows mean amplitudes for each condition and each individual. As predicted, results showed that the overall AAN (across all conditions) was negative $(M=-1.1 \mu \mathrm{V})$. Critically, AAN was lateralized in that the contralateral electrodes were more negative than the ipsilateral ones $\left(M=-0.3 \mu \mathrm{V}, B F_{10}=87.2\right)$. There was weak evidence that this difference (contra minus ipsi) was similar for left and right clicks (interaction $M=0.2$ $\left.\mu \mathrm{V}, 95 \% \mathrm{CI}[-0.1,0.6], B F_{01}=1.7\right)$.

Although the overall LP was positive $(M=1.5 \mu \mathrm{V})$, it did not differ between contralateral and ipsilateral electrodes $(M$ $\left.=-0.1 \mu \mathrm{V}, B F_{10}=0.1 \approx B F_{01}=8.2\right)$. There was moderate evidence that the difference (contra minus ipsi) was similar for left and right clicks (interaction $M=0 \mu \mathrm{V}, 95 \%$ CI [ -0.2 , 0.3], $B F_{01}=6.4$ ).

As seen in Figure $3 \mathrm{C}$, the topographies of AAN and LP did not perfectly match the preregistered electrode selection (as expected). However, exploratory analyses with data-driven electrode selection (separately for AAN and LP) yielded similar results to those with the preregistered electrode selection (see supplementary material; Wiens et al., 2021).

\section{Discussion}

We hypothesized that in hearing, the early NCC (AAN) is lateralized whereas the late NCC (LP) is not. Lateralized binaural clicks were presented at the individual threshold of hearing, and a contrastive analysis was based on each individual's perception of the clicks (aware vs. unaware). Each combination of click side and hemisphere showed clear evidence for AAN and LP. However, only AAN was lateralized in relation to auditory stimulation, that is, it was stronger for contralateral than ipsilateral electrodes. This was supported by very strong evidence $(B F=87)$ for a lateralization of AAN and moderate evidence $(B F=8)$ for no lateralization of LP. Therefore, our findings demonstrate that only the early NCC (but not the late NCC) matches the side of the perceived stimulus in hearing.

The present findings are important because previous research in auditory lateralization could not resolve whether early and late NCC are lateralized (e.g., Butcher et al., 2011; Gutschalk and Steinmann, 2015; Virtanen et al., 1998; Woldorff et al., 1999; Alain et al., 2001; Hautus and Johnson, 2005; Königs and Gutschalk, 2012; Sanders et al., 2008). Most of these studies did not measure awareness, and those that did 


\section{AUDITORY AWARENESS NEGATIVITY IS LATERALIZED}
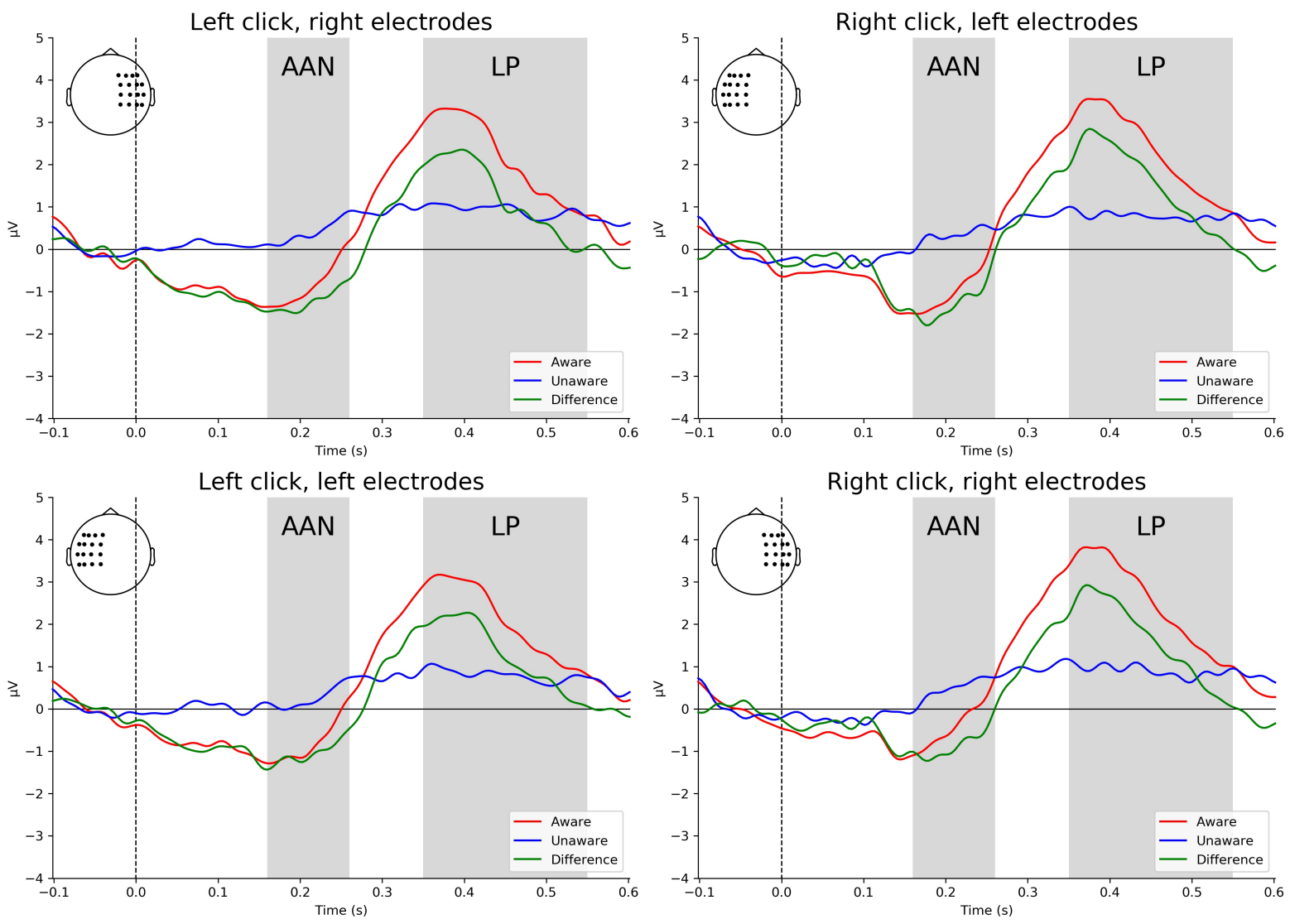

Figure 2: Grand mean $(N=26)$ waves for aware (correct) trials, unaware (correct and incorrect) trials, and their difference (aware minus unaware), separated by click side and electrode laterality. Click side is organized by column, and electrode laterality is organized by row (contralateral on top and ipsilateral on bottom). Auditory awareness negativity (AAN) was measured between 160 and $260 \mathrm{~ms}$ after click onset, and late positivity (LP) was measured between 350 and 550 ms after click onset. These intervals are marked in gray. The data were referenced to the tip of the nose. In the plots, the data were low-pass filtered at $30 \mathrm{~Hz}$.

Table 2

Descriptive and inferential statistics for the mean amplitudes $(\mu \mathrm{V})$ of the difference waves (aware [correct] minus unaware [correct and incorrect])

\begin{tabular}{|c|c|c|c|c|c|c|}
\hline ERP & Condition & $N$ & Mean & $S D$ & $95 \% \mathrm{Cl}$ & $B F_{10}$ \\
\hline \multirow[t]{4}{*}{ AAN } & Overall & 26 & -1.1 & 1.2 & {$[-1.6,-0.6]$} & 574.1 \\
\hline & Contralateral & 26 & -1.3 & 1.1 & {$[-1.7,-0.8]$} & 1609.8 \\
\hline & Ipsilateral & 26 & -1.0 & 1.2 & {$[-1.5,-0.5]$} & 128.3 \\
\hline & Difference & 26 & -0.3 & 0.4 & {$[-0.4,-0.2]$} & 87.2 \\
\hline \multirow[t]{4}{*}{ LP } & Overall & 26 & 1.5 & 0.6 & $1.2,1.7]$ & 2546369.1 \\
\hline & Contralateral & 26 & 1.4 & 0.7 & $1.2,1.7]$ & 2589401.1 \\
\hline & Ipsilateral & 26 & 1.5 & 0.7 & {$[1.2,1.8]$} & 1350426.8 \\
\hline & Difference & 26 & -0.1 & 0.3 & {$[-0.2,0.1]$} & 0.1 \\
\hline
\end{tabular}

Note. The difference condition refers to contralateral minus ipsilateral. AAN = auditory awareness negativity; $L P=$ late positivity; $B F=$ Bayes Factor

lacked a contrastive analysis in which trials rated as aware are contrasted with trials rated as unaware to identify the NCC. In experiments that code trials only on the basis of objective performance, awareness is not isolated (Eklund et al., 2020). Instead, subjective reports are necessary as markers of awareness (Ramsøy and Overgaard, 2004). Accordingly, previous research that did not use a contrastive analysis on the basis of awareness ratings may have identified contralateral auditory processing without demonstrating awarenessrelated auditory lateralization. To avoid this problem, we used a contrastive analysis and stimuli that subjects perceived as lateralized clicks. As the contrastive analysis was conducted for trials that were physically identical, physical stimulus confounds were avoided. Thus, AAN and LP were com- 
A

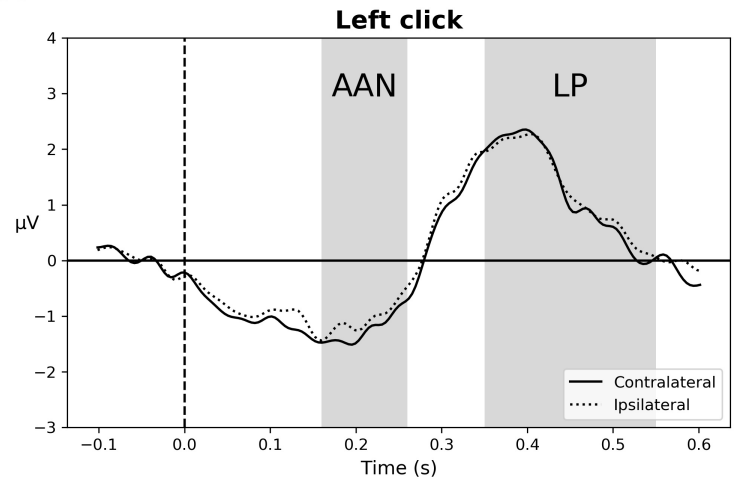

\section{C}

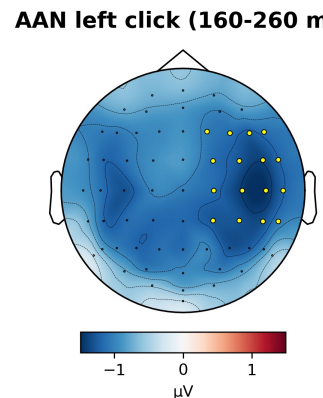

B

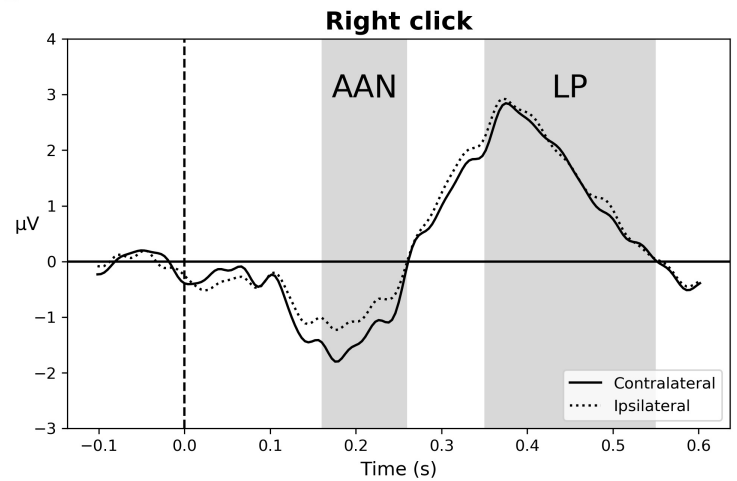

Aanc
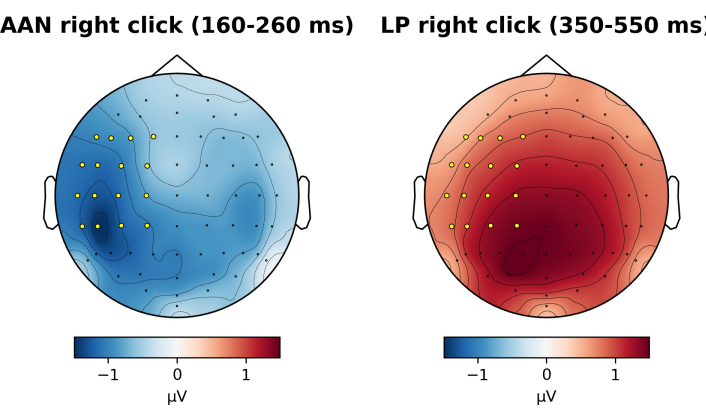

Figure 3: A and B: Grand mean $(N=26)$ difference waves between aware (correct) trials and unaware (correct and incorrect) trials, separately for each click side. A: The response to left clicks from contralateral (right) and ipsilateral (left) electrodes. B: The response to right clicks from contralateral (left) and ipsilateral (right) electrodes. Auditory awareness negativity (AAN) was measured between 160 and 260 ms after click onset, and late positivity (LP) was measured between 350 and 550 ms after click onset. These intervals are marked in gray. C: Topographies of mean amplitudes for AAN and LP for left and right clicks (preregistered electrodes are marked). The data were referenced to the tip of the nose. In the plots, the data were low-pass filtered at $30 \mathrm{~Hz}$.

puted to physically identical clicks. Furthermore, in contrast to previous research, AAN and LP were measured to both the left and right side.

We showed that lateralized clicks were associated with a lateralized AAN. Because the topography of the AAN changed in relation to the perceived location of the sound (left or right), this finding supports the idea that AAN is an index of the conscious perception of lateralized sounds. Importantly, similar results have been reported for vision and touch. In vision, lateralized (left or right) visual field stimulation results in a stronger contralateral than ipsilateral visual awareness negativity (Eklund and Wiens, 2018; Koivisto and Grassini, 2016). Similarly, in touch, lateralized (left arm) sensory stimulation results in awareness-related activity in the right somatosensory cortex (N140; Auksztulewicz and Blankenburg, 2013; Forschack et al., 2020). Because the neural sources of the early NCC have been found to be localized to sensory areas (Eklund and Wiens, 2019; Koivisto and Revonsuo, 2010), these findings in combination strongly suggest that these different versions of the early NCC capture processes that are similar in function even though they may originate from different sensory areas (Dembski et al., 2021).

Although AAN is a correlate of awareness, the present results do not resolve whether AAN is an index of aware- ness or either a prerequisite or consequence of awareness (Aru et al., 2012; de Graaf et al., 2012). The global neuronal workspace theory suggests that early sensory activity is preconscious and that only late global activity mediates consciousness (Dehaene et al., 2006). From this perspective, AAN (as the early NCC) indexes preconscious processing (i.e., it is a prerequisite) rather than consciousness per se. Accordingly, it is irrelevant for the global neuronal workspace theory that AAN is lateralized whereas LP (the late NCC) is not. Nonetheless, because our findings show that AAN is lateralized whereas LP is not, they imply that AAN and LP capture separate aspects of consciousness. At face validity, AAN is a more suitable NCC than LP because it correlates more closely with lateralized experiences. Furthermore, because LP varies with other phenomena such as response manipulations, it does not appear to be a pure index of awareness but is also affected by the consequences of consciousness (Pitts et al., 2018; Tsuchiya et al., 2015; Förster et al., 2020; Dembski et al., 2021).

Strictly speaking, our findings cannot support recurrent processing theory over the global neuronal workspace theory. Nonetheless, we suggest that AAN is an indirect index of recurrent processing, as suggested by research in other sensory modalities (Auksztulewicz and Blankenburg, 2013; 

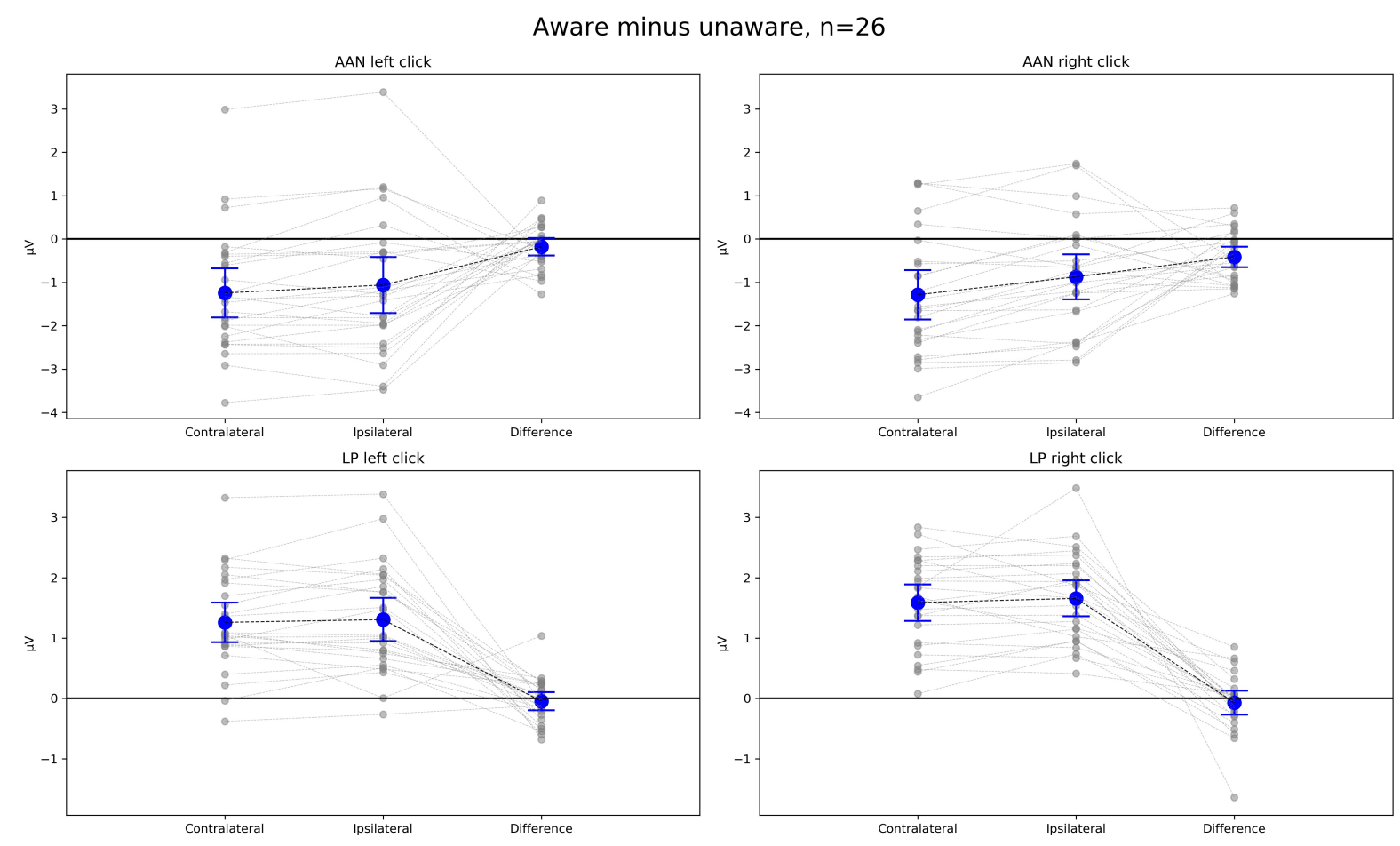

Figure 4: Mean amplitudes $(N=26)$ for the AAN-relevant electrodes and interval (top row) and for the LP-relevant electrodes and interval (bottom row), separately for left clicks (left column) and right clicks (right column). Electrodes were either ipsilateral to the click side or contralateral to the click side. The difference refers to contralateral minus ipsilateral. The data were referenced to the tip of the nose.

Boehler et al., 2008). In the visual domain, Boehler et al. (2008) showed that contralateral visual recurrent processing correlates with visual awareness. The authors compared visual targets rated as aware with targets rated as unaware as neural activity was recorded with magnetoencephalography. Feedforward and feedback activity was spatiotemporally tracked along the visual hierarchy. Only feedback activity from higher visual areas to the contralateral primary visual area between 110 to $170 \mathrm{~ms}$ after target onset correlated with reported awareness of the visual targets. In the somatosensory domain, Auksztulewicz and Blankenburg (2013) showed that contralateral recurrent processing correlates with awareness. The authors stimulated the left median nerve at the subject's detection threshold while recording EEG. Dynamic causal modeling suggested that recurrent processing in the right somatosensory cortex $140 \mathrm{~ms}$ after stimulus onset correlated with reported awareness of the somatosensory stimulation. Taken together, these findings are consistent with recurrent processing theory (Lamme, 2006): Feedback from hierarchically higher sensory areas to primary sensory areas enables awareness of sensory content. In our study, the timing of AAN (after about $100 \mathrm{~ms}$ ) corresponds well to the findings in vision (Boehler et al., 2008) and in the somatosensory modality (Auksztulewicz and Blankenburg, 2013), as it matches the expected interval of recurrent processing. However, because we did not track the feedforward and feedback activity, future research needs to examine whether AAN reflects recurrent processing.

In conclusion, Bayesian analyses provided very strong evidence that the early correlate of auditory awareness (AAN) is stronger for contralateral than ipsilateral electrodes and moderate evidence that the late correlate of auditory awareness (LP) is not lateralized. These findings demonstrate that the early but not the late neural correlate of auditory awareness reflects lateralized experiences. Therefore, our findings support that AAN and LP capture separate aspects of consciousness.

\section{A. Declarations}

\section{A.1. Acknowledgement}

The authors thank Steve Palmer for editing an earlier version of this manuscript.

\section{A.2. Funding}

This work was supported by Marianne and Marcus Wallenberg Foundation (Grant 2019-0102).

\section{A.3. Conflict of interest statement}

The authors declare that the research was conducted in the absence of any commercial or financial relationships that could be construed as a potential conflict of interest. 


\section{AUDITORY AWARENESS NEGATIVITY IS LATERALIZED}

\section{A.4. Ethical approval and consent to participate and publish}

The experiment adhered to the declaration of Helsinki. Participants provided their written informed consent to participate in this study and that their raw data will be shared anonymized.

\section{A.5. Open practices statement}

The data and materials for all experiments and analyses are available at a university repository (Wiens et al., 2021).

This study was preregistered at OSF (https://doi.org/ 10.17605/OSF. IO/PSRJF).

\section{CRediT authorship contribution statement}

Rasmus Eklund: Designed the study, programmed the experiment, collected the data, wrote the scripts to analyze the behavioral and EEG data, and wrote the manuscript. Billy Gerdfeldter: Designed the study, collected the data, and wrote the manuscript. Stefan Wiens: Designed the study and wrote the manuscript.

\section{References}

Alain, C., Arnott, S.R., Picton, T.W., 2001. Bottom-up and Top-down Influences on Auditory Scene Analysis: Evidence from Event-Related Brain Potentials. Journal of Experimental Psychology: Human Perception and Performance 27, 1072-1089. doi:10.1037/0096-1523.27.5.1072.

Andersen, L.M., Pedersen, M.N., Sandberg, K., Overgaard, M., 2016. Occipital MEG activity in the early time range $(<300 \mathrm{~ms})$ predicts graded changes in perceptual consciousness. Cerebral Cortex 26, 2677-2688. doi:10.1093/cercor/bhv108.

Appelhoff, S., Sanderson, M., Brooks, T.L., van Vliet, M., Quentin, R., Holdgraf, C., Chaumon, M., Mikulan, E., Tavabi, K., Höchenberger, R., Welke, D., Brunner, C., Rockhill, A.P., Larson, E., Gramfort, A., Jas, M., 2019. MNE-BIDS: Organizing electrophysiological data into the BIDS format and facilitating their analysis. Journal of Open Source Software 4, 1896. doi:10.21105/joss. 01896.

Aru, J., Bachmann, T., Singer, W., Melloni, L., 2012. Distilling the neural correlates of consciousness. Neuroscience \& Biobehavioral Reviews 36, 737-746. doi:10.1016/j. neubiorev. 2011.12.003.

Auksztulewicz, R., Blankenburg, F., 2013. Subjective Rating of Weak Tactile Stimuli Is Parametrically Encoded in Event-Related Potentials. Journal of Neuroscience 33, 11878-11887. doi:10.1523/JNEUROSCI. 4243-12. 2013.

Boehler, C.N., Schoenfeld, M.A., Heinze, H.J., Hopf, J.M., 2008. Rapid Recurrent Processing Gates Awareness in Primary Visual Cortex. Proceedings of the National Academy of Sciences 105, 8742-8747. doi:10. 1073/pnas. 0801999105.

Butcher, A., Govenlock, S.W., Tata, M.S., 2011. A Lateralized Auditory Evoked Potential Elicited When Auditory Objects Are Defined by Spatial Motion. Hearing Research 272, 58-68. doi:10.1016/j. heares. 2010. 10.019.

Crick, F., Koch, C., 1990. Towards a neurobiological theory of consciousness. Seminars in the neurosciences 2, 263-275.

de Graaf, T.A., Hsieh, P.J., Sack, A.T., 2012. The 'Correlates' in Neural Correlates of Consciousness. Neuroscience \& Biobehavioral Reviews 36, 191-197. doi:10.1016/j. neubiorev. 2011.05.012.

Dehaene, S., Changeux, J.P., Naccache, L., Sackur, J., Sergent, C., 2006. Conscious, preconscious, and subliminal processing: A testable taxonomy. Trends in Cognitive Sciences 10, 204-211. doi:10.1016/j.tics. 2006.03.007.

Dembski, C., Koch, C., Pitts, M., 2021. Physiological Correlates of Sensory Consciousness: Evidence for a Generalized Awareness Negativity doi:10.31234/osf.io/ma36g.
Dienes, Z., McLatchie, N., 2018. Four reasons to prefer bayesian analyses over significance testing. Psychonomic Bulletin \& Review 25, 207-218. doi:10.3758/s13423-017-1266-z.

Dykstra, A.R., Cariani, P.A., Gutschalk, A., 2017. A Roadmap for the Study of Conscious Audition and Its Neural Basis. Phil. Trans. R. Soc. B 372, 20160103. doi:10.1098/rstb. 2016.0103.

Eklund, R., Gerdfeldter, B., Wiens, S., 2019. Effects of a manual response requirement on early and late correlates of auditory awareness. Frontiers in Psychology 10. doi:10.3389/fpsyg. 2019.02083.

Eklund, R., Gerdfeldter, B., Wiens, S., 2020. Is auditory awareness negativity confounded by performance? Consciousness and Cognition 83, 102954. doi:10.1016/j. concog. 2020.102954.

Eklund, R., Wiens, S., 2018. Visual awareness negativity is an early neural correlate of awareness: A preregistered study with two gabor sizes. Cognitive, Affective, \& Behavioral Neuroscience 18, 176-188. doi:10.3758/s13415-018-0562-z.

Eklund, R., Wiens, S., 2019. Auditory awareness negativity is an electrophysiological correlate of awareness in an auditory threshold task. Consciousness and Cognition 71, 70-78. doi:10.1016/j. concog. 2019.03.008.

Forschack, N., Nierhaus, T., Müller, M.M., Villringer, A., 2020. Dissociable Neural Correlates of Stimulation Intensity and Detection in Somatosensation. NeuroImage 217, 116908. doi:10.1016/j. neuroimage. 2020.116908.

Förster, J., Koivisto, M., Revonsuo, A., 2020. ERP and MEG correlates of visual consciousness: The second decade. Consciousness and Cognition 80, 102917. doi:10.1016/j. concog. 2020.102917.

Gramfort, A., Luessi, M., Larson, E., Engemann, D.A., Strohmeier, D., Brodbeck, C., Goj, R., Jas, M., Brooks, T., Parkkonen, L., Hämäläinen, M., 2013. MEG and EEG Data Analysis with MNE-Python. Frontiers in Neuroscience 7, 1-13. doi:10.3389/fnins.2013.00267.

Gramfort, A., Luessi, M., Larson, E., Engemann, D.A., Strohmeier, D., Brodbeck, C., Parkkonen, L., Hämäläinen, M.S., 2014. MNE Software for Processing MEG and EEG Data. NeuroImage 86, 446-460. doi:10. $1016 / j$. neuroimage. 2013.10 .027$.

Gutschalk, A., Steinmann, I., 2015. Stimulus Dependence of Contralateral Dominance in Human Auditory Cortex: Contralateral Dominance in Auditory Cortex. Human Brain Mapping 36, 883-896. doi:10.1002/ hbm. 22673.

Hautus, M.J., Johnson, B.W., 2005. Object-Related Brain Potentials Associated with the Perceptual Segregation of a Dichotically Embedded Pitch. The Journal of the Acoustical Society of America 117, 275-280. doi: $10.1121 / 1.1828499$.

Keil, A., Debener, S., Gratton, G., Junghöfer, M., Kappenman, E.S., Luck, S.J., Luu, P., Miller, G.A., Yee, C.M., 2014. Committee Report: Publication Guidelines and Recommendations for Studies Using Electroencephalography and Magnetoencephalography. Psychophysiology 51,121. doi:10.1111/psyp. 12147.

Koivisto, M., Grassini, S., 2016. Neural Processing Around 200 ms After Stimulus-Onset Correlates with Subjective Visual Awareness. Neuropsychologia 84, 235-243. doi:10.1016/j. neuropsychologia.2016.02. 024.

Koivisto, M., Revonsuo, A., 2010. Event-Related Brain Potential Correlates of Visual Awareness. Neuroscience \& Biobehavioral Reviews 34, 922 934. doi:10.1016/j. neubiorev. 2009.12.002.

Königs, L., Gutschalk, A., 2012. Functional Lateralization in Auditory Cortex Under Informational Masking and in Silence. European Journal of Neuroscience 36, 3283-3290. doi:10.1111/j.1460-9568.2012.08240.x.

Lamme, V.A.F., 2006. Towards a true neural stance on consciousness. Trends in Cognitive Sciences 10, 494-501. doi:10.1016/j.tics.2006. 09.001.

Luck, S.J., Gaspelin, N., 2017. How to get statistically significant effects in any ERP experiment (and why you shouldn't). Psychophysiology 54, 146-157. doi:10.1111/psyp. 12639.

Meyer, K., 2011. Primary Sensory Cortices, Top-down Projections and Conscious Experience. Progress in Neurobiology 94, 408-417. doi:10. 1016/j. pneurobio.2011.05.010.

Nosek, B.A., Ebersole, C.R., DeHaven, A.C., Mellor, D.T., 2018. The preregistration revolution. Proceedings of the National Academy of Sci- 
ences 115, 2600-2606. doi:10.1073/pnas.1708274114.

Peirce, J.W., 2007. PsychoPy-Psychophysics Software in Python. Journal of Neuroscience Methods 162, 8-13. doi:10.1016/j.jneumeth.2006.11. 017.

Pernet, C.R., Appelhoff, S., Gorgolewski, K.J., Flandin, G., Phillips, C., Delorme, A., Oostenveld, R., 2019. EEG-BIDS, an extension to the brain imaging data structure for electroencephalography. Scientific Data 6, 103. doi:10.1038/s41597-019-0104-8.

Pickles, J.O., 2015. Chapter 1 - Auditory pathways: Anatomy and physiology, in: Aminoff, M.J., Boller, F., Swaab, D.F. (Eds.), Handbook of Clinical Neurology. Elsevier. volume 129 of The Human Auditory System, pp. 3-25. doi:10.1016/B978-0-444-62630-1.00001-9.

Pitts, M.A., Lutsyshyna, L.A., Hillyard, S.A., 2018. The Relationship Between Attention and Consciousness: An Expanded Taxonomy and Implications for 'no-Report' Paradigms. Phil. Trans. R. Soc. B 373, 20170348. doi:10.1098/rstb. 2017.0348.

Ramsøy, T.Z., Overgaard, M., 2004. Introspection and Subliminal Perception. Phenomenology and the Cognitive Sciences 3, 1-23. doi:10.1023/ B:PHEN.0000041900.30172.e8.

Sandberg, K., Timmermans, B., Overgaard, M., Cleeremans, A., 2010. Measuring Consciousness: Is One Measure Better Than the Other? Consciousness and Cognition 19, 1069-1078. doi:10.1016/j.concog. 2009.12.013.

Sanders, L.D., Joh, A.S., Keen, R.E., Freyman, R.L., 2008. One Sound or Two? Object-Related Negativity Indexes Echo Perception. Perception \& Psychophysics 70, 1558-1570. doi:10.3758/PP.70.8.1558.

Snyder, J.S., Yerkes, B.D., Pitts, M.A., 2015. Testing Domain-General Theories of Perceptual Awareness with Auditory Brain Responses. Trends in Cognitive Sciences 19, 295-297. doi:10.1016/j.tics.2015.04.002.

Thaler, L., Reich, G.M., Zhang, X., Wang, D., Smith, G.E., Tao, Z., Abdullah, R.S.A.B.R., Cherniakov, M., Baker, C.J., Kish, D., Antoniou, M., 2017. Mouth-Clicks Used by Blind Expert Human Echolocators - Signal Description and Model Based Signal Synthesis. PLOS Computational Biology 13, e1005670. doi:10.1371/journal.pcbi.1005670.

Tsuchiya, N., Wilke, M., Frässle, S., Lamme, V.A.F., 2015. No-Report Paradigms: Extracting the True Neural Correlates of Consciousness. Trends in Cognitive Sciences 19, 757-770. doi:10.1016/j.tics. 2015. 10.002.

Virtanen, J., Ahveninen, J., Ilmoniemi, R.J., Näätänen, R., Pekkonen, E., 1998. Replicability of MEG and EEG Measures of the Auditory N1/N1m-Response. Electroencephalography and Clinical Neurophysiology/Evoked Potentials Section 108, 291-298. doi:10.1016/ s0168-5597 (98)00006-9.

Wagenmakers, E.J., Love, J., Marsman, M., Jamil, T., Ly, A., Verhagen, J., Selker, R., Gronau, Q.F., Dropmann, D., Boutin, B., Meerhoff, F., Knight, P., Raj, A., van Kesteren, E.J., van Doorn, J., Šmíra, M., Epskamp, S., Etz, A., Matzke, D., de Jong, T., van den Bergh, D., Sarafoglou, A., Steingroever, H., Derks, K., Rouder, J.N., Morey, R.D., 2018. Bayesian inference for psychology. Part II: Example applications with JASP. Psychonomic Bulletin \& Review 25, 58-76. doi:10.3758/s13423-017-1323-7.

Wagenmakers, E.J., Morey, R.D., Lee, M.D., 2016. Bayesian benefits for the pragmatic researcher. Current Directions in Psychological Science 25, 169-176. doi:10.1177/0963721416643289.

Wiens, S., Eklund, R., Gerdfeldter, B., 2021. Open data: The early but not the late neural correlate of auditory awareness is contralateral to auditory stimulation. Stockholm: Stockholm University doi:10.17045/sthlmuni. 13067018.

Woldorff, M.G., Tempelmann, C., Fell, J., Tegeler, C., Gaschler-Markefski, B., Hinrichs, H., Heinze, H.J., Scheich, H., 1999. Lateralized Auditory Spatial Perception and the Contralaterality of Cortical Processing as Studied with Functional Magnetic Resonance Imaging and Magnetoencephalography. Human Brain Mapping 7, 49-66. doi:10.1002/(SICI) 1097-0193 (1999) 7: 1<49: : AID-HBM5>3 . 0. CO;2-J. 